\title{
Guide Sign Analysis of Traffic Sign Data-Set Using Supervised Spiking Neuron Technique
}

\author{
Mohd Safirin Karis ${ }^{1,3 *}$, Nursabillilah Mohd Ali ${ }^{1,3}$, Mohd Azamuddin Ali ${ }^{1,3}$, Muhamad Raimi Sadiq Samsudin ${ }^{1,3}$, \\ Nurasmiza Selamat ${ }^{1,3}$, Wira Hidayat Mohd Saad ${ }^{1,3}$, Amar Faiz Zainal Abidin ${ }^{2,3}$, Zairi Ismael Rizman ${ }^{4}$ \\ ${ }^{I}$ Faculty of Electrical Engineering, Universiti Teknikal Malaysia Melaka (UTeM), Durian Tunggal, Melaka, Malaysia \\ ${ }^{2}$ Faculty of Engineering Technology, Universiti Teknikal Malaysia Melaka (UTeM), Durian Tunggal, Melaka, Malaysia \\ ${ }^{3}$ Centre of Robotics and Industrial Automation, Universiti Teknikal Malaysia Melaka (UTeM), Durian Tunggal, Melaka, Malaysia \\ ${ }^{4}$ Faculty of Electrical Engineering, Universiti Teknologi MARA, 23000 Dungun, Terengganu, Malaysia \\ *Corresponding author E-mail: safirin@utem.edu.my
}

\begin{abstract}
In this paper, 20 guided traffic signs mostly displayed around Malacca area were selected as project databased. Early hypothesis was made as the error for each usable image will increased as more interference introduced to the original image used. Three types of conditions which are hidden region, image brightness and image rotation were selected as an experiment to analyze the performance of each sign used. Each condition will perform a specific error to generate their mean value and in the same, image recognition will take place in the matchup process. By focusing on the result, it produces hidden region critically ascending mean error value at $62.5 \%=0.07$ and has average value at others points. For image brightness effect, it shows a higher mean error value collected at less brightness points and nonstable pattern at $10 \%$ to $60 \%$ brightness. As for rotation upshot, the values show a critically ascending for error value at $22.5 \%$ and slightly increase at $2 \%$ to $5 \%$ rotation point. For the recognition process, at $6.25 \%$ hidden region, almost $70 \%$ of images are correctly matched to its own classes while at $62.5 \%$ hidden region only $40 \%$ of images are correctly matched to its own classes and leaving 2 images to outperform. For $-40 \%$ brightness, $45 \%$ of images are correctly matched to its own classes while at $60 \%$ brightness $65 \%$ of images are correctly matched to its own classes and leaving 1 image to outperform. Lastly, at 2.5 degree rotation, $85 \%$ of images are correctly matched to its own classes while at $25^{\circ}$ rotation, $45 \%$ of images are correctly matched to its own classes and leaving 2 images to outperform. Finally, the error forms will affect the final output response of the detected traffic signs used.
\end{abstract}

Keywords: SNN; traffic sign; hidden region; brightness; rotation; mean error; detection; recognition.

\section{Introduction}

Artificial neural networks it's all about understanding the early computations that take place in the dense networks connected neurons cell in central nervous system of human being. Originally, in 1943, McCulloch and Pitts proposed a model in view of improved double neurons, where a solitary neuron actualizes a basic thresholding function, a neuron's state is either dynamic or not dynamic, and this is controlled by ascertaining the weighted entirety of the conditions of neurons it is associated with [1]. Spiking neural system (SNN) which is named the third era of neural system had extremely well structures, suited for complex data handling. Creatures demonstrate an extraordinary simplicity as a team with element situations, raising enthusiasm for the utilization of simulated neural systems in undertakings that arrangement with real-world interaction. Throughout the years, three generation of simulated neural systems have been proposed, each generation naturally more reasonable and computationally more powerful [1]. Spiking neural systems utilize the component of time in conveying by conveying singular heartbeats. Spiking neurons can in this manner multiplex data into a solitary stream of signs, similar to the recurrence and adequacy of sound in the sound-related framework.

Because of the ascent in number of vehicle around the world, street mischance has turned out to be one of the real reasons for fatalities. It is anticipated to be the fifth leading cause for death in the world 2030. In Malaysia, street activity wounds were accounted for to be the fifth leading cause for death. As said, fatal accidents in 2001 were reported have 5856 death compared to 6200 death in 2005 [5]. They do not believe they got caught. It's common for some drivers to think there are little chances of them being caught by police for ignoring traffic signs and signals. It is also leads by nowadays advanced technology of Global Positioning System (GPS), whereas the system always can detect where the speed limit or trap designed by the police-man on highways. Even though the system was designed to turned drivers to be polite in road, some of them easily use it in bad manners. This type of attitude can easily lead to an accident that gives injury or kills innocent people. Previously, many works focused on intelligent application for image processing research [8-12].

As purposed, SNN will give fast response to assist road user to detect incoming traffic sign. The main objective for this project is to study the relationship between SNN and traffic signs whether it is suitable to achieve the goals of fast reaction. Artificial Intelligence commonly describe how traffic signs detection works with Convolutional Neural Network and also extremely proven by many researcher with the assistance of machine learning such as Adaboost, Support Vector Machine (SVM), PHOG, HOG, DLReSuMe and many more. Now, by the development of SNN, the application of detection can surely bring the world of automation into a new valley of advanced technology. 


\section{Traffic Sign}

Traffic sign are vital part of the highway system. Traffic signs are the oldest and most used traffic control devices on the road. Traffic sign provide a means of communicating important information about the roadway to the driver. Traffic signs utilize colour, shape, symbols and/or words to convey information.

Without traffic sign, the rate of road accidents may increase. This is because the road drivers often violate the rules of the road even when warning signs are placed. With this traffic sign, it can reduce the number of traffic accidents and facilitate mobility and can make the community more integrated with road rules. This is because, if we see not only adults who use roads, children, seniors, teens and even people with disabilities are also using the road.

The traffic sign will not be placed without any reason, the party should see various angles and aspects of putting signboards according to size and colour. Traffic sign is divided into several colours like red, blue, green and also yellow. Each colour plays a different role. Apart from colour, traffic sign also has different forms according to the suitability of the place and condition.

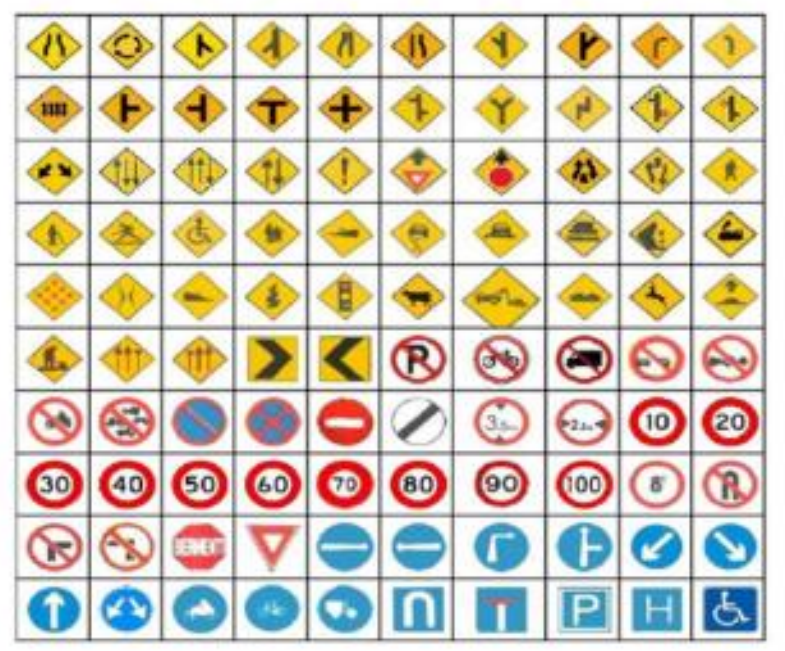

Fig. 1: Symbol of traffic sign [6]

The red means that it is forbidden to the driver while the blue colour is a guide to the distance and the yellow colour gives a warning as shown in Figure 2. The fact is that many people still do not know the meaning of this colour even though the traffic sign has existed for so long.

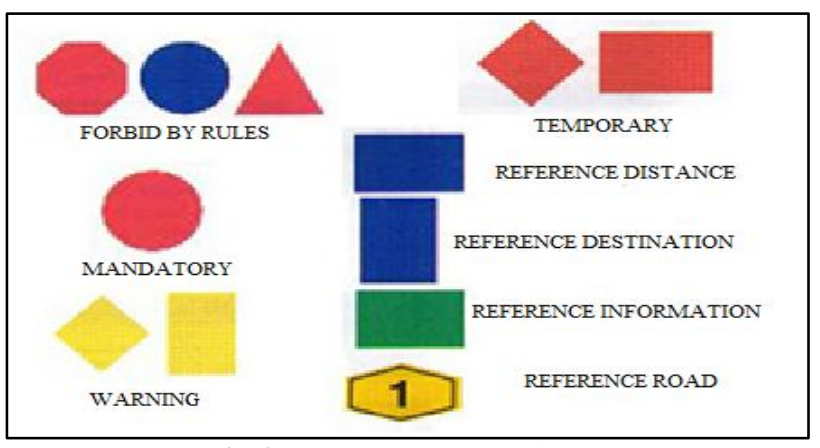

Fig. 2: Characteristics of traffic sign [6]

In Malaysia, child road traffic injuries (age 0-19 years old) account for $35.5,35.3$, and $35.2 \%$ of total admission to Ministry of Health hospitals due to road crashes in 2003, 2004 and 2005 respectively. They are the top leading cause of hospital admission among children (0-19 years) due to injuries in Malaysia for 20032005 (Figure 3). Figure 3 shows that the number of traffic injuries recorded is twice the number of injuries due to a fall among children in Malaysia aged 0-19 years [13].

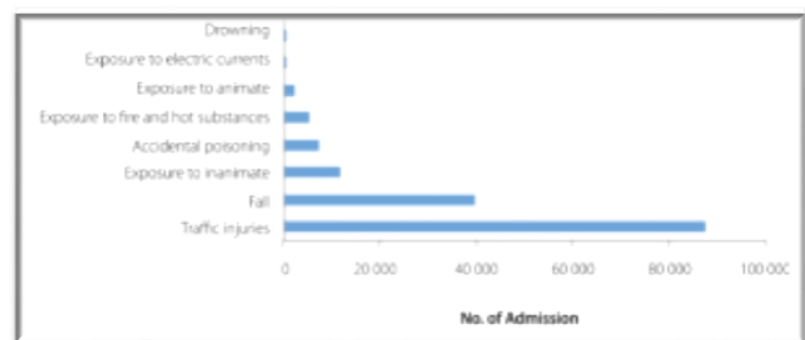

Fig. 3: Child road traffic injuries [7]

So, traffic sign is very important because it is very helpful in reducing the rate of road accidents. Indirectly, traffic sign is related to the project that will be conducting on the guide sign and warning sign.

\section{Model}

A simple spiking model equation (1) and (2) are presented as biologically plausible as the Hodgkin-Huxley model [4], yet as computationally efficient as the integrate-and-fire model by the combination experimental studies of animal and human nervous systems with numerical simulation of huge scale brain models relying on four parameters $\mathrm{a}, \mathrm{b}, \mathrm{c}$ and $\mathrm{d}$. The model produced spiking and bursting behavior of known types of cortical neurons, as illustrated in Figure 4. The derivation of first (1) equation is based on bifurcation theory and ordinary form reduction [4].

Here, $\mathrm{v}$ and $\mathrm{u}$ are dimensionless variables, and $\mathrm{a}, \mathrm{b}, \mathrm{c}$ and $\mathrm{d}$ are dimensionless parameter and ' $=d / d t$, where $\mathrm{t}$ is the time. The variable $v$ represents the membrane potential of the neuron and $u$ represents a membrane recovery parameter, which accounts for the activation of $\mathrm{K}+$ ionic currents and inactivation of $\mathrm{Na}+$ ionic currents, and it provides negative feedback to $v$.

After the spike reaches its apex $(+30 \mathrm{mV})$, the membrane voltage and the recovery variable are reset according to equation (3). Synaptic currents or injected DC-currents are delivered to variable $I$. Value of $I$ currently were set with each output data values of each image. Figure 1 shows sample of output spikes type where the FS is the most compatible reaction concomitant with the project objectives.
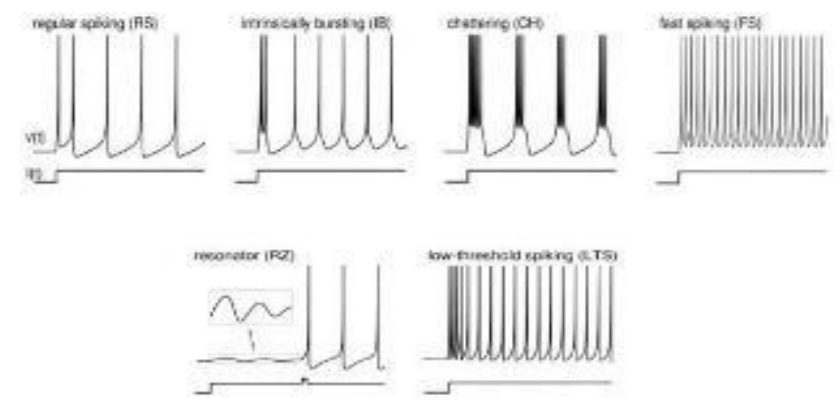

Fig. 4: Type of dynamic response [4]

Euler's Method of 2-D system with first order differential equation:

$\mathrm{v}^{\prime}=0.04 \mathrm{v}^{2}+5 \mathrm{v}+140-\mathrm{u}+I$

$\mathrm{u}^{\prime}=\mathrm{a}^{*}(\mathrm{bv}-\mathrm{u})$

After spike-reset:

$v \geq 30=\left\{\begin{array}{c}v=c \\ u=u+d\end{array}\right.$

where a-time scale for recovery variable $\mathrm{u}, \mathrm{b}$-Sensitivity for recovery variable $\mathrm{v}, \mathrm{c}$-After spikes reset value of membrane potential $\mathrm{v}$ and d-After spikes reset value of membrane potential $\mathrm{u}$. 


\section{Architecture}

\subsection{SNN Architecture for Clustering Images}

The model for a spiking neuron which was used in the following is the spike response model with short term memory. Consideration of a network of such spiking engineering in a completely associated feed forward with associations executed as numerous deferred synaptic terminals as shown in Figure 5.

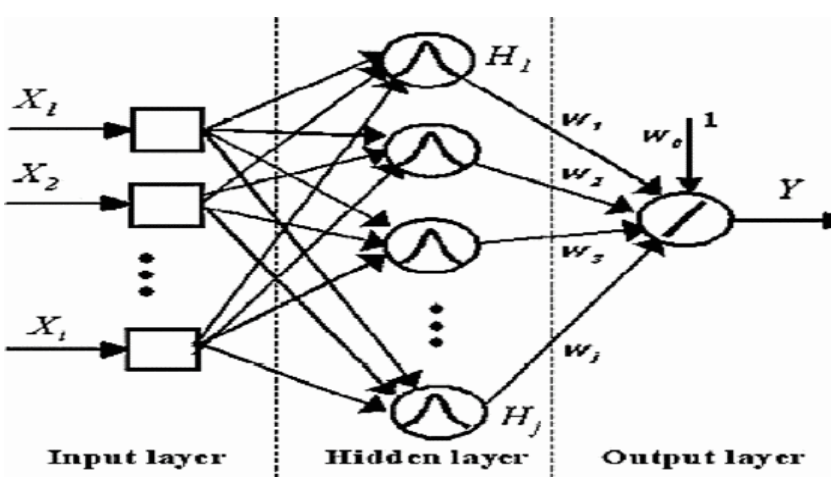

Fig. 5: Segmentation and clustering image [1]

\subsection{SNN Architecture for Cell Segmentation}

In this part, two basic terms can be highlighted. First, the image processing technique using spiking neuron for shape of nuclei from microscopic color images was spotted. Second, the increasing number of cancer screening which make the quantity of information that pathologist must dealing with will larger. In this case, the microscopic cellular image should be in good output condition to produce the best and reliable of the analysis result outcome.

Cellular segmentation is widely introduced nowadays. They in clude watershed, region-based and threshold-based methods. Classification of cells can be divide into three parts, which are background, cytoplasm and nucleus [3].

Furthermore, its cannot be perfect as a fraction on nuclei pixels will have same color with the pixels from the cytoplasm because of the nuclei habits according to the type of cells and the distribution of chromatin. In addition, for some topologies, the mucus appears in the background has the same color as the other cells such as nucleus and cytoplasm.

\subsection{SNN Architecture for Edge Detection}

To begin, picture of a minute cell is portioned with spiking neural system. Once the division done, the recording process will take place as it will record the movement of every yield neuron which gives every info pixel a yield twofold 1 if the neuron is dynamic or 0 if the neuron is idle [1]. The after effect of two fold networks actuation of yield neurons can be spoken by pairing the pictures containing the edges recognized by these neurons for each of their classes. Next, the combination is done to obtain the last edges by superimposing the subsequent pictures.

\section{Architecture}

In the process of developing the spike response, the equation of $\mathrm{v}$ $=0.04 \mathrm{v}^{2}+5 \mathrm{v}+140-\mathrm{u}+I$ need to attach to the MATLAB coding. The equation was obtained by fitting the spike initiation so that the membrane potential $v$ has $m V$ scale and the time $t$ has $m s$ scale. The resting potential in the model is between -70 and $-60 \mathrm{mV}$ depending on the value of $b$. Most of the origin neurons modelling do not have a fixed threshold, depending on the history of the membrane potential prior to the spike. The threshold potential can be as low as $-55 \mathrm{mV}$ or as high as $-40 \mathrm{mV}$.

\subsection{Training with Neural Network Toolbox}

In order to perform the supervised learning algorithm such as proposed, training data for recognition part is another step to perform the recognition. In this system, all the output data projected by the spiking coding as to classify its classes. In this research the scope will be focusing on 20 number of most displayed guided traffic sign around Malacca area. All the traffic signs used were listed as in Table 1 .

Table 1: Image classes

\begin{tabular}{|l|l|}
\hline \multicolumn{2}{|c|}{ Table 1: Image classes } \\
\hline 1) Airport & 11) Turn Left \\
\hline 3) Gicycle Lane & 12) Turn Right \\
\hline 4) Go Right & 13) One way \\
\hline 5) Keep Left & 14) Parking \\
\hline 6) Keep Right & 15) Dead End \\
\hline 7) Keep Right & 16) Hospital \\
\hline 8) Keep Straight or Left & 17) Disabilities \\
\hline 9) Keep Straight Or Right & 18) Petrol Station \\
\hline 10) U-turn & 19) Mosque \\
\hline
\end{tabular}

From the above table, all the sign are referred to blue sign which are the guide sign used in Malaysia. To perform the neural network, MATLAB2013a is used to develop the classification for each image and the errors to analyze the output results. A folder of an input and target need to be specified first in order to perform the neural activity.

The network keep to train until the regression plot achieved its stability when $\mathrm{Y}=\mathrm{T}$ signify the network was successfully trained correctly.

\subsection{Detection and Recognition}

To perform the output result, all the input images were put together in a specific parent folder. Each of them was classified into their own each target. After that, through the detection part, blob analysis block diagram is connected to perform rectangle bound as making it a specific detection around the detected traffic sign.

\section{Spike Response Output}

The 20 traffic signs were used to perform spike response. Each figure shows the comparison of voltage change at receptor 10 and receptor 200. Table 2 shows the output from spike response.

Table 1: Image perform spike response

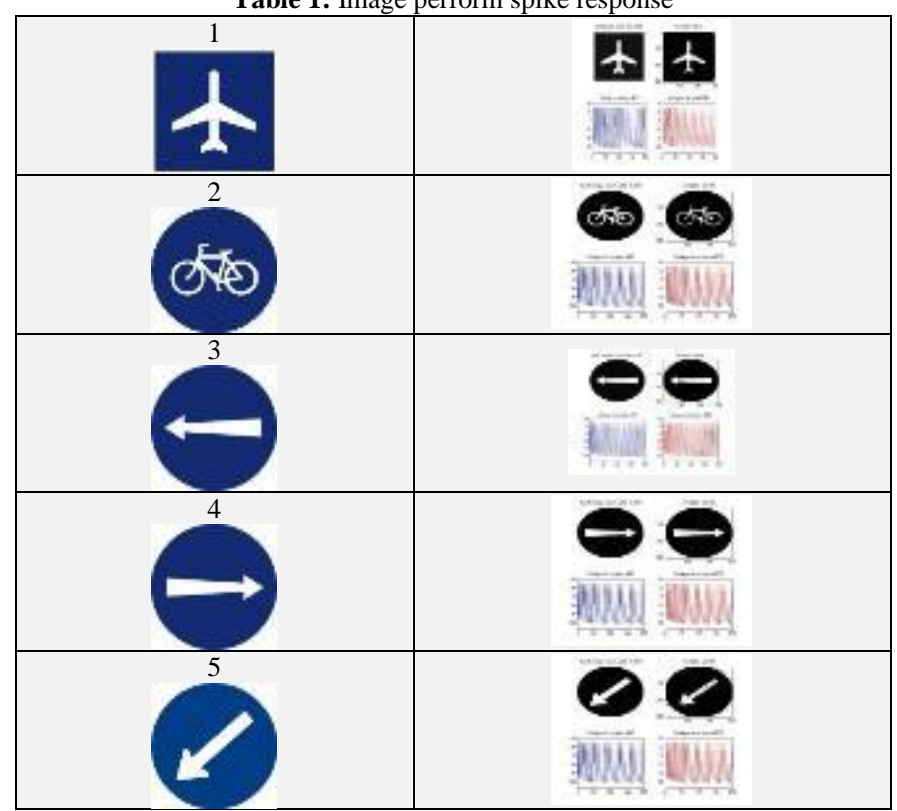




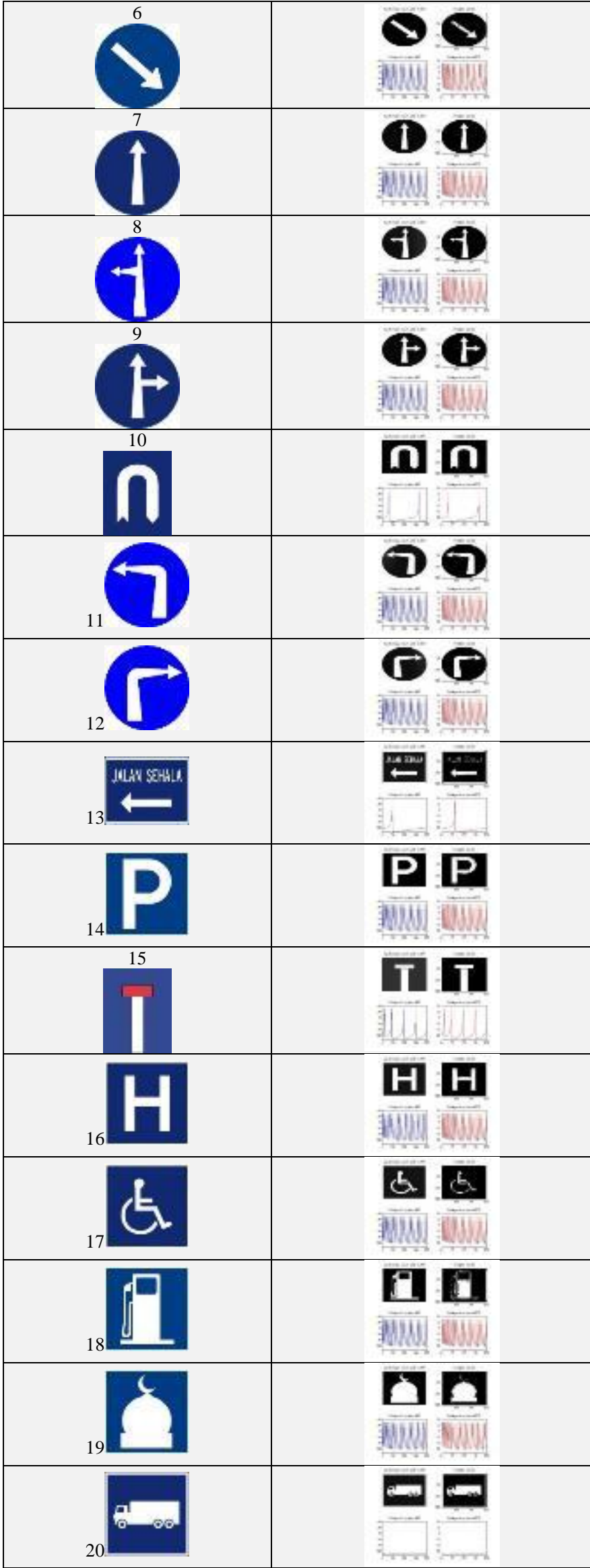

Table 2 shows the spikes response from each image input. After doing an observation, it can be found that almost every image performed the low-threshold spiking (LTS) type. U-turn, One Way and Dead End traffic sign has performed regular spiking (RS) type while at Truck Stops sign has performed an abnormality of resonator (RZ) type.

Low-threshold Spiking, LTS is the inhibitory cortical cells. Neurons fire high-frequency through the action potentials, but with a noticeable spike frequency slowing down. These neurons have low firing thresholds, which affects the value of $b=0.25$ in the model.

Regular Spiking, RS neurons are the most typical neurons in the outer layer of cells. When activate with long stimulus the neurons fire a few spikes between all of them and then the period increases. The frequency decreases. Increasing the strength of the injected DC-current increases the spike between neurons frequency, though it never becomes too fast because of large spike-after hyperpolarization. In this implemented model, its consequence the value of $c$ and $d$. Value of $\mathrm{d}$ will be higher because of the pulses jump of $u$ and affects the value of $c=-65 \mathrm{mV}$ had made the voltage value resets for each cooldown.

Resonator, RZ neurons have damped or supported subthreshold motions. They resonate to input sources which having suitable frequency. This character can leads to value $a$ becomes 0.1 and leads value $b$ to 0.26 .

After performed the spike response, the project continues to perform image classification to its own classes. These errors are generated from the non-real time analysis to obtain their error values.

\section{Mean Error Recognition}

After performed the spike response, the project continues to perform image classification to its own classes. In here, there are three types of conditions which are hidden region, image brightness and image rotation. An analysis can be conducted to compare each image error through their specific condition. Each condition will perform a specific error value, then the value of each image through their own condition will sum up to generate the mean value as we can see in section 7.1, 7.2 and 7.3 below.

\subsection{Experiment 1: Hidden Region Effect}

This experiment is to determine the effect of hidden region toward image recognition. The guided sign used is purposely hiding begins with $6.25 \%, 12.5 \%, 18.75 \%, 25 \%, 31.25 \%, 37.5 \%, 43.75 \%$, $50 \%, 56.25 \%$ and $62.5 \%$ to see the error produce for each sign in system recognition. The mean error is collected for every sign used in every hidden region shown in Figure 6.

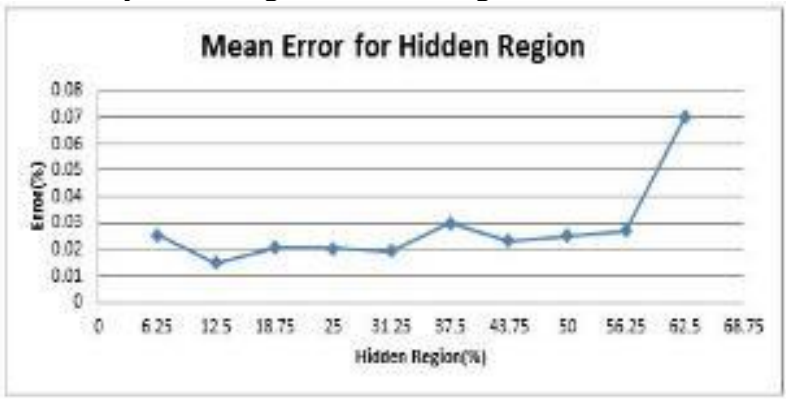

Fig. 6: Mean error for hidden region

Based on the mean error for hidden region graph above, the values shows a critically ascending for error value at $62.5 \%$ hidden region equal to 0.07 and has average value at others points. Thus, the hypothesis was accepted as the value of hidden region coverage will influence the increasing value of error.

\subsection{Experiment 2: Brightness Effect}

In this experiment, the brightness of the sign used being varied. Several points were collected included $-40,-30,-20,-10,10,20$, $30,40,50$ and 60 to see its effect. The mean error for each reading were recorded as shown in Figure 7. 


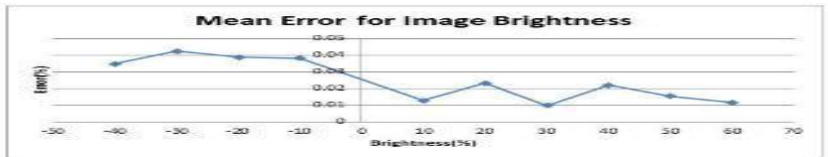

Fig. 7: Mean error for brightness

Based on the mean error for image brightness graph above, the data shows a higher value collected at less brightness (darker) points and non-stable pattern at $10 \%$ to $60 \%$ brightness. Thus, the hypothesis was accepted where the value of image brightness affected the increasing value of error.

\subsection{Experiment 3: Rotational Effect}

For this experiment, rotation for each sign used is purposely introduced. It begins with $2.5^{\circ}, 5^{\circ}, 7.5^{\circ}, 10^{\circ}, 12.5^{\circ}, 15^{\circ}, 17.5^{\circ}, 20^{\circ}, 22.5^{\circ}$ and $25^{\circ}$ to see the rotational effects towards the image recognition of this system. The mean error graph was listed and shown in Figure 8.

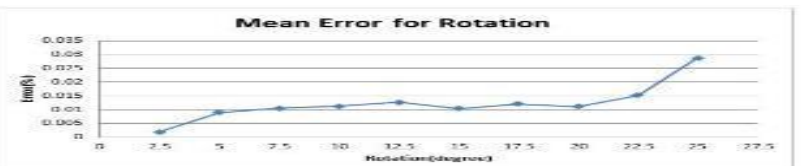

Fig. 8: Mean error for rotation

Based on the mean error for rotation graph above, the values shows a critically ascending for error value at $22.5 \%$ and slightly increase at $2 \%$ to $5 \%$ rotation point. Thus, the hypothesis was accepted where the higher image rotation, higher error value produced by each image.

\section{Output Image Recognition}

An analysis to form display image also been conducted throughout this project. In this part, the lowest and the highest value for each image condition for hidden region, brightness and rotational were observed. To recognize each image input to its output, the output value of basic image after training process need to sum up with the output error of each image after training process. Table 3, Table 4 and Table 5 are used to give a reference to analyze the output image produced.

\subsection{Hidden Region}

Table 3: Comparison image output and input for hidden region

\begin{tabular}{|c|c|c|c|}
\hline \multicolumn{2}{|c|}{$6.25 \%$ Hidden Region } & \multicolumn{2}{c|}{$62.5 \%$ Hidden Region } \\
\hline $\begin{array}{c}\text { Input Image } \\
\text { (Class) }\end{array}$ & $\begin{array}{c}\text { Output Image } \\
\text { (Class) }\end{array}$ & $\begin{array}{c}\text { Input Image } \\
\text { (Class) }\end{array}$ & $\begin{array}{c}\text { Output Image } \\
\text { (Class) }\end{array}$ \\
\hline 1 & $1^{*}$ & 1 & 8 \\
\hline 2 & 7 & 2 & $2^{*}$ \\
\hline 3 & 4 & 3 & 6 \\
\hline 4 & 9 & 4 & 7 \\
\hline 5 & $5^{*}$ & 5 & Out of range \\
\hline 6 & $6^{*}$ & 6 & $6^{*}$ \\
\hline 7 & 14 & 7 & $7^{*}$ \\
\hline 8 & $8^{*}$ & 8 & 13 \\
\hline 9 & $9^{*}$ & 9 & $9^{*}$ \\
\hline 10 & $10^{*}$ & 10 & 12 \\
\hline 11 & $11^{*}$ & 11 & 14 \\
\hline 12 & 13 & 12 & $12^{*}$ \\
\hline 13 & 17 & 13 & 17 \\
\hline 14 & $14^{*}$ & 14 & Out of range \\
\hline 15 & $15^{*}$ & 15 & $15^{*}$ \\
\hline 16 & $16^{*}$ & 16 & 13 \\
\hline 17 & $17^{*}$ & 17 & $17^{*}$ \\
\hline 18 & $18^{*}$ & 18 & 11 \\
\hline 19 & $19^{*}$ & 19 & $19^{*}$ \\
\hline 20 & $20^{*}$ & 20 & 10 \\
\hline
\end{tabular}

To summarized, at $6.25 \%$ hidden region, almost $70 \%$ of images are correctly matched to its own classes while at $62.5 \%$ hidden region, $40 \%$ of images are correctly matched to its own classes and leave 2 images to performed as out of range (no recognition process).

\subsection{Brightness}

Table 4: Comparison image output and input for brightness

\begin{tabular}{c|c|c|c}
\hline \multicolumn{2}{c}{$-40 \%$ Brightness } & \multicolumn{2}{c}{$60 \%$ Brightness } \\
\hline $\begin{array}{c}\text { Input Image } \\
\text { (Class) }\end{array}$ & $\begin{array}{c}\text { Output Image } \\
\text { (Class) }\end{array}$ & $\begin{array}{c}\text { Input Image } \\
\text { (Class) }\end{array}$ & $\begin{array}{c}\text { Output Image } \\
\text { (Class) }\end{array}$ \\
\hline 1 & 11 & 1 & $1^{*}$ \\
2 & 13 & 2 & 4 \\
3 & 12 & 3 & $3^{*}$ \\
4 & 10 & 4 & 7 \\
5 & 13 & 5 & 6 \\
6 & 12 & 6 & $6^{*}$ \\
7 & $7^{*}$ & 7 & $7^{*}$ \\
8 & $8^{*}$ & 8 & $8^{*}$ \\
9 & $9^{*}$ & 9 & $10^{*}$ \\
10 & 9 & 10 & $11^{*}$ \\
11 & $11^{*}$ & 11 & $12^{*}$ \\
12 & $12^{*}$ & 12 & 15 \\
13 & 17 & 13 & 4 \\
14 & 16 & 14 & $15^{*}$ \\
15 & 1 & 15 & $16^{*}$ \\
16 & $16^{*}$ & 16 & 19 \\
17 & $17^{*}$ & 17 & $18^{*}$ \\
18 & $18^{*}$ & 18 & Out of range \\
19 & $19^{*}$ & 19 & $20^{*}$ \\
20 & 16 & 20 & \\
\hline
\end{tabular}

At $-40 \%$ brightness $45 \%$ of images are correctly matched to its own classes while at $60 \%$ brightness $65 \%$ of images are correctly matched to its own classes and 1 image performed an out of range (no recognition process).

\subsection{Rotational}

Table 5: Comparison image output and input for rotation

\begin{tabular}{|c|c|c|c|}
\hline \multicolumn{2}{|c|}{$6.25 \%$ Rotation } & \multicolumn{2}{c|}{$62.5 \%$ Rotation } \\
\hline $\begin{array}{c}\text { Input Image } \\
\text { (Class) }\end{array}$ & $\begin{array}{c}\text { Output Image } \\
\text { (Class) }\end{array}$ & $\begin{array}{c}\text { Input Image } \\
\text { (Class) }\end{array}$ & $\begin{array}{c}\text { Output Image } \\
\text { (Class) }\end{array}$ \\
\hline 1 & 8 & 1 & 9 \\
\hline 2 & $2^{*}$ & 2 & 5 \\
\hline 3 & $3^{*}$ & 3 & $3^{*}$ \\
\hline 4 & 7 & 4 & 7 \\
\hline 5 & $5^{*}$ & 5 & 6 \\
\hline 6 & $6^{*}$ & 6 & $6^{*}$ \\
\hline 7 & $7^{*}$ & 7 & $7^{*}$ \\
\hline 8 & $8^{*}$ & 8 & 9 \\
\hline 9 & $9^{*}$ & 9 & 10 \\
\hline 10 & $10^{*}$ & 10 & $10^{*}$ \\
\hline 11 & $11^{*}$ & 11 & 5 \\
\hline 12 & $12^{*}$ & 12 & $12^{*}$ \\
\hline 13 & 2 & 13 & $13^{*}$ \\
\hline 14 & $14^{*}$ & 14 & Out of range \\
\hline 15 & $15^{*}$ & 15 & $15^{*}$ \\
\hline 16 & $16^{*}$ & 16 & Out of range \\
\hline 17 & $17^{*}$ & 17 & 18 \\
\hline 18 & $18^{*}$ & 18 & $18^{*}$ \\
\hline 19 & $19^{*}$ & 19 & $19^{*}$ \\
\hline 20 & $20^{*}$ & 20 & 10 \\
\hline
\end{tabular}

For 2.5 degree rotation, $85 \%$ of images are correctly matched to its own classes. While at 25 degree rotation, $45 \%$ of images are correctly matched to its own classes and 2 images performed an out of range (no recognition process).

\section{Conclusion and Recommendations}

In conclusion, Spiking Neuron equation and theories (currently used the simplest model) were successfully implemented in this 
project. Ultimately, the project was developed well and the data analyses were successfully collected. Thus, the objective was achieved.

\section{Acknowledgement}

The authors would like to thanks Universiti Teknikal Malaysia Melaka (UTeM), Faculty of Electrical Engineering (FKE) and Centre of Robotics and Industrial Automation (CeRIA) for their financial support.

\section{References}

[1] B. Meftah, O. Lézoray, S. Chaturvedi, A. A. Khurshid, and A Benyettou, "Image processing with spiking neuron networks," Stud. Comput. Intell., vol. 427, (2013), pp. 525-544.

[2] S. Bohte (2003), Spiking neural networks.

[3] A. Grüning and S. M. Bohte, "Spiking Neural Networks: Principles and Challenges," Elen.Ucl.Ac.Be, no. April, pp. 23-25, 2014

[4] Eugene M. Izhikevich "Simple Model of Spiking Neurons" (PDF).Vol 14, No. 6, Dated December 2003.

[5] M. M. Lau, K. H. Lim, and A. A. Gopalai, "Malaysia traffic sign recognition with convolutional neural network," 2015 IEEE Int. Conf. Digit. Signal Process, (2015), pp. 1006-1010.

[6] http://www.safetysign.com/road-symbol-signs.

[7] https://www.mendeley.com/research-papers/road-safety-planmalaysia-

20062010/?utm_source=desktop\&utm_medium $=1.17 .12 \& u t m \_c a m$ paign=open_catalog\&userDocumentId=\%7B89de605b-4cd4-427a9f3d-38d1a78c7244\%7D.

[8] N Mohd Ali, MS Karis, SA Ahmad Tarusan, Gao-Jie Wong, MS Mohd Aras, MB Bahar, AF Zainal Abidin, "Inspection and Quality Checking of Ceramic Cup using Machine Vision Technique: Design and Analysis", Jurnal Teknologi, (2017), pp. 33-38.

[9] N Mohd Ali, MS Karis, NM Mohd Sobran, MB Bahar, Oh Kok Ken, M Mat Ibrahim, NF Johan, "Detection of Multiple Mangoes using Histogram of Oriented Gradient Technique in Aerial Monitoring", ARPN Journal of Engineering and Applied Sciences, (2017), pp. 2730-2736.

[10] MS Karis, N Mohd Ali, A Mohd Basar, HI Jaafar, AF Zainal Abidin, "An Analysis on out-of-plane Face Detection among Female Student and Illumination effects using SIFT and SUFT", AIP Conference Proceedings, (2016).

[11] MS Karis, N Mohd Ali, WH Mohd Saad, AF Zainal Abidin, N Ismaun, M Abd Aziz, "Performance Analysis between Keypoints of SURF and Skin Colour YCBCR based Technique for Face Detection among Final Year UTeM Male Student", Jurnal Teknologi (2017), pp. 65-69.

[12] N Mohd Ali, MS Karis, AF Zainal Abidin, B Bakri, NR Abd Razif, "Traffic Sign Detection and Recognition: Review and Analysis", Jurnal Teknologi, (2017), pp. 107-113.

[13] Mohamed N, Voon WS, Hashim HH, Othman I (2011), An overview of road traffic injuries among children in Malaysia and its implication on road traffic injury prevention strategy. https://www.researchgate.net/profile/Hizal_Hanis_Hashim/publicat on/312771185_Research_report_an_overview_of_road_traffic_inju ries_among_children_in_Malaysia_and_its_implication_on_road_tr

fic_injury_prevention_strategy/links/5a8508db4585159152b814ea/ Research-report-an-overview-of-road-traffic-injuries-amongchildren-in-Malaysia-and-its-implication-on-road-traffic-injuryprevention-strategy.pdf. 